\title{
Having More Healthy Practice was Associated with Low White Blood Cell Counts in Middle- aged Japanese Male and Female Workers
}

\author{
Rei OTSUKA ${ }^{1,2}$, Koji TAMAKOSHI ${ }^{1}$, Keiko WADA ${ }^{1}$, Kunihiro MATSUSHITA ${ }^{1,3}$, \\ Pei OUYANG ${ }^{1}$, Yo HOTTA ${ }^{1,4}$, Seiko TAKEFUJI ${ }^{1,4}$, Hirotsugu MITSUHASHI ${ }^{1,3}$, \\ Hideaki TOYOSHIMA ${ }^{1,5}$, Hiroshi SHIMOKATA ${ }^{2}$ and Hiroshi YATSUYA ${ }^{1}$ \\ ${ }^{1}$ Department of Public Health/ Health Information Dynamics, Field of Social Life Science, Nagoya University \\ Graduate School of Medicine, 65 Tsurumai-cho, Showa-ku, Nagoya 466-8550, Japan \\ ${ }^{2}$ Department of Epidemiology, National Institute for Longevity Sciences, National Center for Geriatrics and \\ Gerontology, Obu, Japan \\ ${ }^{3}$ Department of Cardiology, Nagoya University Graduate School of Medicine, Nagoya, Japan \\ ${ }^{4}$ Department of Metabolic Diseases, Nagoya University Graduate School of Medicine, Nagoya, Japan \\ ${ }^{5}$ Health Care Center of Anjo Kosei Hospital, Aichi, Japan
}

Received December 28, 2007 and accepted April 14, 2008

\begin{abstract}
White blood cell (WBC) count is well known to be an independent risk marker for cardiovascular disease. The aim of this study is to examine the relationships of WBC counts to seven health practices including obesity, eating habits, smoking, alcohol intake, sleeping, physical activity, and perceived mental stress, and then clustering the relevant healthy practices. The subjects were 1,492 male and 316 female Japanese workers aged $40 \mathrm{yr}$ and over in 2002. Each of seven health practices from a self-administered questionnaire was categorized as a 'healthy' or 'unhealthy' practice, and WBC counts from fasting blood samples were determined by automated particle counters. The means of age and WBC counts were $49.5 \mathrm{yr}$ and $5,375 \mathrm{cells} / \mu \mathrm{l}$ in men, and $48.6 \mathrm{yr}$ and $4,890 \mathrm{cells} / \mu \mathrm{l}$ in women, respectively. After multivariate adjustments for all health practices and age, the estimated $\mathrm{WBC}$ counts were significantly lower in normal weight subjects and never or former smokers $(p<0.01)$. Age-adjusted WBC counts decreased significantly by $204.9 \pm 23.7$ cells $/ \mu$ l (means \pm SE) and $117.6 \pm 53.2$ cells $/ \mu$ l for each increase in one healthy practice $(p<0.05)$, respectively, suggesting that cultivating healthier practices would lead to lower WBC counts. This study recommends modifying unhealthy practice one by one and maintaining healthy practices as an effective strategy for the prevention of atherosclerotic diseases, in addition, to quit smoking or abstain from heavy smoking especially in men is important to prevent the low-grade inflammation.
\end{abstract}

Key words: White blood cell, Healthy practice, Cross-sectional study, Questionnaire, Epidemiology, Japanese

\section{Introduction}

The white blood cell (WBC) count is an objective marker of acute infection, tissue damage, and other inflammatory, immunological, or hematological conditions ${ }^{1,2)}$. Many epidemiological studies have implicated

*To whom correspondence should be addressed.
WBC counts as an independent risk marker for cardiovascular disease (CVD) and have suggested that WBC may be important in the development and progression of atherosclerosis and cardiovascular disease ${ }^{3-6)}$.

From the viewpoint of primary prevention of atherosclerotic disease, it is important to examine the associations between WBC counts and some lifestyles among apparently healthy men since such lifestyles have been 
thought to be related to an increase in WBC counts ${ }^{7-11)}$. Previous epidemiological studies have suggested an association between elevated WBC counts and low physical activity $^{7,8)}$, poor sleep ${ }^{9)}$, and smoking ${ }^{10,11)}$. However, the evidence of an association between WBC and other basic lifestyles is sparse ${ }^{12,13)}$. In addition, it is not clear whether the clustering of healthy practices within individuals as measured by the number of healthy practices is associated with WBC counts.

Therefore, we analyzed the relationships of WBC counts with seven health practices including obesity, eating habits, smoking, alcohol intake, sleeping, physical activity, and perceived mental stress, and examined the clustering the relevant healthy practices in a large sample of Japanese men and women in the workplace.

\section{Materials and Methods}

\section{Study subjects}

A survey was conducted in 2002 of local government offices in Aichi Prefecture, central Japan. The survey included a self-administered questionnaire and a physical examination including height and weight. WBC counts were available only among participants in health checkups. Among them, those who expressed their written consent to the use of information on lifestyle and from physical examinations numbered 1,515 men and 317 women.

Six health practices including obesity, eating habits, smoking status, alcohol intake, sleeping hours, and physical activity were selected as study variables from physical examinations and self-administered questionnaires with reference to seven health practices proposed by Breslow ${ }^{14)}$, in addition to which we added perceived mental stress. All health practices except for obesity were asked about the past month.

We excluded participants with WBC counts of $10,000 \geq$ cells $/ \mu$ ( 23 men and 1 woman), indicating a clinically relevant inflammatory condition. Finally, the current analysis was restricted to 1,492 men and 316 women with complete data on all the lifestyle components and WBC counts. All subjects signed an informed consent statement, and this study was approved by the Ethics Review Committee of the Nagoya University Graduate School of Medicine, Nagoya, Japan.

Biochemical analysis and anthropometric measurements

Venous whole blood samples were drawn from an antecubital vein after the subjects fasted for $8 \mathrm{~h}$ or overnight. WBC counts were determined by automated particle counters within $24 \mathrm{~h}$ after venipuncture in a commercial laboratory.

Height and weight were measured when subjects were dressed in light indoor clothing and without footwear.
Height in $\mathrm{cm}$ was measured to the nearest $0.1 \mathrm{~cm}$, and weight in $\mathrm{kg}$ was measured to the nearest $0.1 \mathrm{~kg}$. Body mass index (BMI) was calculated as weight in kilograms divided by the square of the height in meters.

\section{Statistical analysis}

All analyses were performed separately for men and women. Each of seven health practices was categorized into two or three groups, and then divided into 'healthy' and 'unhealthy' practices with reference to seven health practices proposed by Breslow ${ }^{14}$ as follows:

1. Obesity: 'normal weight $\left(<25 \mathrm{~kg} / \mathrm{m}^{2}\right)$ ' and 'obese $\left(\geq 25 \mathrm{~kg} / \mathrm{m}^{2}\right)$ ' according to the classification of the Japan Society for the Study of Obesity ${ }^{15)}$.

2. Eating habits: Subjects were to reply either 'Yes' or 'No' to the question: 'Do you usually pay attention to your eating habits?'

3. Smoking status: 'former and never smoker' or 'current smoker'.

4. Alcohol intake: ' $<23$ g' and ' $\geq 23$ g' ethanol per day. Alcohol intake evaluated as a subject's usual weekly intake of alcohol was converted to grams of ethanol per day.

5. Sleep: ' $\leq 7-<9$ h' and ' $<7$ or $\leq 9$ h' per day.

6. Physical activity: Subjects were to reply either 'Yes' or 'No' to the question: 'Do you usually try to have physical activity?'

7. Perceived mental stress: 'very much or much' and 'ordinary or little' to the question: 'Do you have much stress in your life?'

Comparisons of WBC counts among each health practice were conducted by $t$-test or analysis of variance (ANOVA). One-way analysis of covariance (ANCOVA) was used to test differences in WBC counts across each health practice after adjustment for covariates including age as a continuous variable and other health practices. These variables with three categories were entered into the model using dummy variables (Table 1). Differences in proportions were tested using $\chi^{2}$ test. The total number of healthy practices was obtained by adding each of the current healthy practices. A linear trend test was also performed by a polynomial contrast procedure in a general linear model (Table 2).

Multiple regression analysis was performed to estimate the contribution of healthy practice to WBC counts, which were taken as the dependent variable, while the independent variables included age and the total number of healthy practices $(0-7)$ (Table 3$)$. All subjects were classified into four groups by the total number of healthy practices, i.e. 0 to $3,4,5$, and 6 to 7 , since only a few subjects had a lower or higher total number of healthy practices.

$p$ values of less than 0.05 were considered statistically 
Table 1. White blood cell counts (cells/ $\mu \mathrm{l})$ according to age and health practices

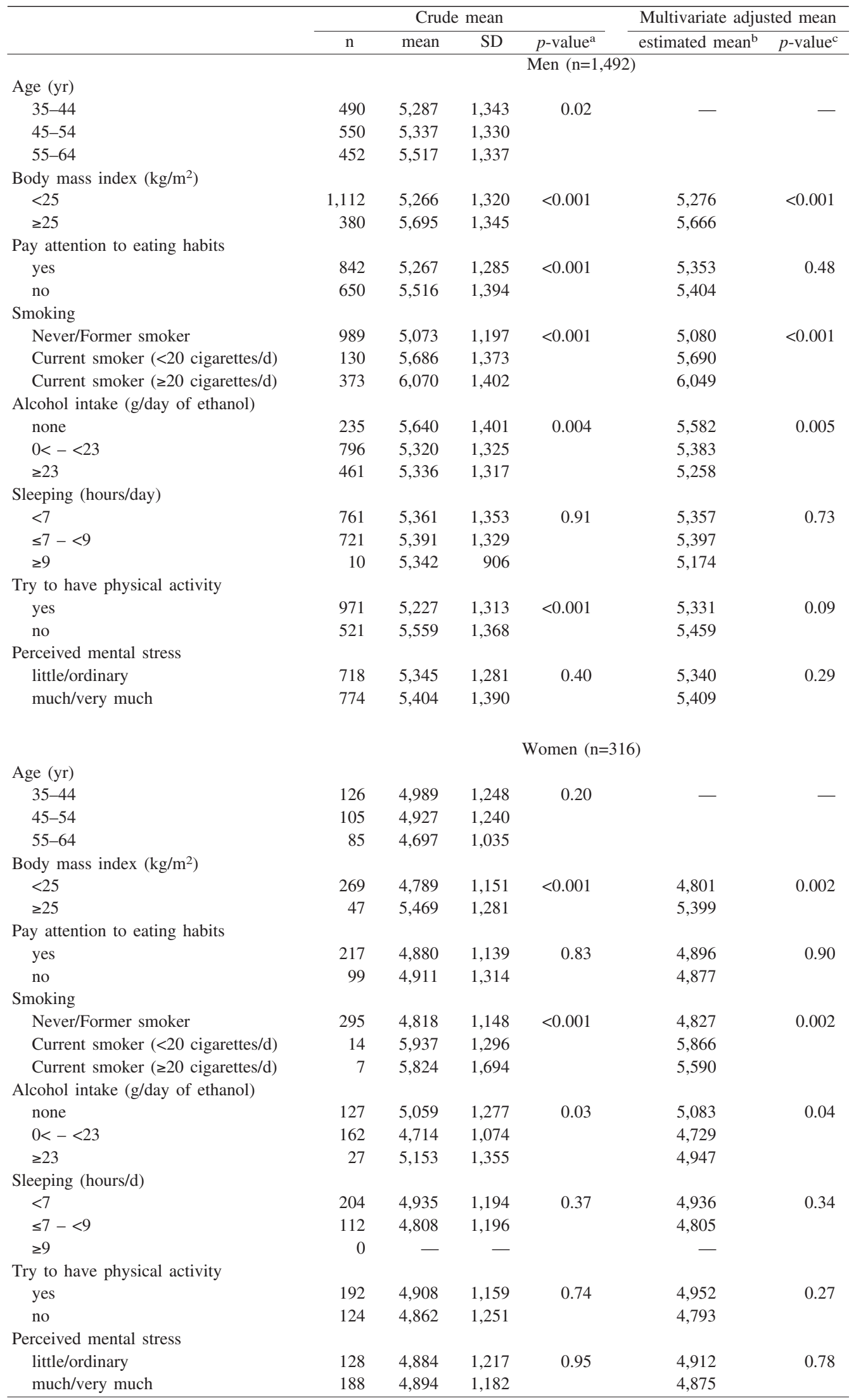

SD: Standard deviations, ${ }^{\mathrm{a}} t$-test ( 2 groups) or one way analyses of variance ( 3 groups), ${ }^{\mathrm{b}}$ Adjusted for age and other health practices, ${ }^{\mathrm{C} O n e}$ way analyses of covariance. 
Table 2. White blood cell counts (cells/ $\mu \mathrm{l})$ according to the total number of seven healthy practices

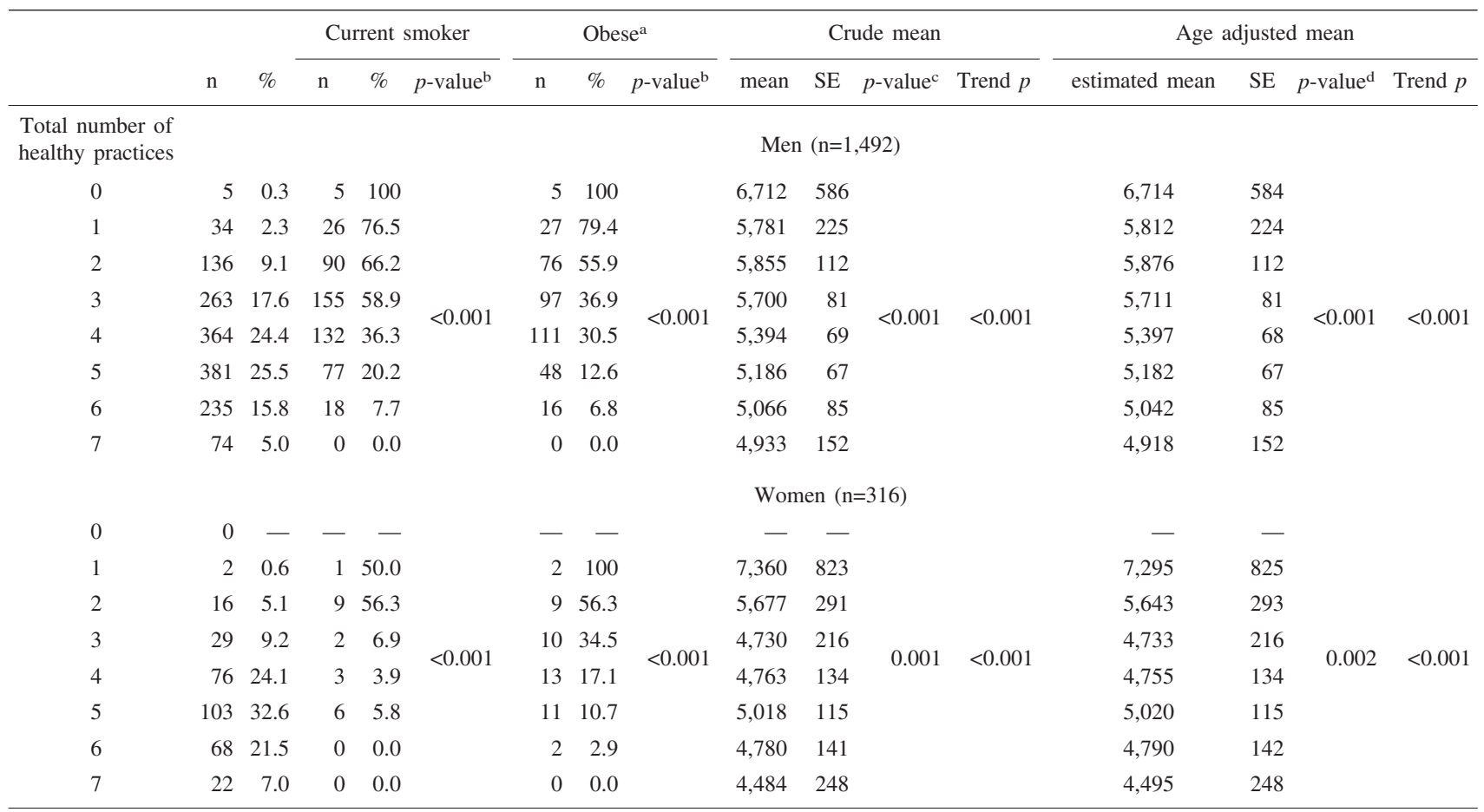

SE: Standard error, ${ }^{\mathrm{a} O b e s e}$ : Body mass index $\geq 25\left(\mathrm{~kg} / \mathrm{m}^{2}\right),{ }^{\mathrm{b}} \chi^{2}$ test, ${ }^{\mathrm{c}}$ One way analyses of variance, ${ }^{\mathrm{d}}$ One way analyses of covariance.

Table 3. Age-adjusted white blood cell counts (cells/ $\mu \mathrm{l}$ ) according to the total number of seven healthy practices and multiple linear regression analysis of WBC counts

\begin{tabular}{|c|c|c|c|c|c|c|c|c|c|}
\hline & \multicolumn{6}{|c|}{ Age-adjusted white blood cell counts } & \multicolumn{3}{|c|}{ Regression coefficients ${ }^{\mathrm{a}}$} \\
\hline & $\mathrm{n}$ & $\%$ & estimated mean & SE & $p$-value ${ }^{\mathrm{b}}$ & Trend $p$ & Age-adjusted means & SE & $p$-value \\
\hline Total number of healthy practices & & & \multicolumn{7}{|c|}{ Men $(n=1,492)$} \\
\hline $0-3$ & 438 & 29.4 & 5,782 & 63 & \multirow{4}{*}{$<0.001$} & \multirow{4}{*}{$<0.001$} & \multirow{4}{*}{-204.9} & \multirow{5}{*}{23.7} & \multirow{4}{*}{$<0.001$} \\
\hline 4 & 364 & 24.4 & 5,397 & 68 & & & & & \\
\hline 5 & 381 & 25.5 & 5,182 & 67 & & & & & \\
\hline $6-7$ & 309 & 20.7 & 5,013 & 75 & & & & & \\
\hline & & & \multicolumn{6}{|c|}{ Women $(n=316)$} & \\
\hline $0-3$ & 47 & 14.9 & 5,147 & 173 & \multirow{4}{*}{0.10} & \multirow{4}{*}{0.13} & \multirow{4}{*}{-117.6} & \multirow{4}{*}{53.2} & \multirow{4}{*}{0.03} \\
\hline 4 & 76 & 24.1 & 4,752 & 136 & & & & & \\
\hline 5 & 103 & 32.6 & 5,021 & 117 & & & & & \\
\hline $6-7$ & 90 & 28.5 & 4,722 & 125 & & & & & \\
\hline
\end{tabular}

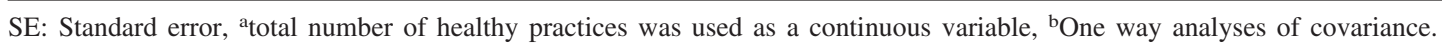

significant. All statistical analyses were conducted using the SPSS statistical package for Windows, version 12.0 (SPSS Inc., Chicago, IL, USA).

\section{Results}

The means (standard deviations: SD) of age, current BMI and WBC counts were 49.5 (6.7) yr, $23.4(2.8) \mathrm{kg} / \mathrm{m}^{2}$ and 5,375 (1339) cells/ $\mu \mathrm{l}$, respectively, in men, and 48.6 (6.6) $\mathrm{yr}$ and $22.2(2.8) \mathrm{kg} / \mathrm{m}^{2}$ and 4,890 (1194) cells $/ \mu \mathrm{l}$, in women.
Table 1 shows the means and differences in WBC counts according to age and health practices. Crude WBC counts were lower in normal weight subjects and never or former smokers among both men and women. Current smokers with more than 20 cigarettes per day had higher WBC counts than those with less than 20 cigarettes per day. The men who paid attention to eating habits also had significantly lower WBC counts $(p<0.001, t$-test). After multivariate adjustment for all these factors including age, the estimated WBC counts remained significantly lower in normal weight subjects $\left(<25 \mathrm{~kg} / \mathrm{m}^{2}\right)$ and never 
or former smoker than in obese subjects $\left(\geq 25 \mathrm{~kg} / \mathrm{m}^{2}\right)$ and current smokers, respectively, both in men and women. On the other hand, WBC counts were lower in men with a daily ethanol intake of $23 \mathrm{~g}$ and over than in men with less than $23 \mathrm{~g}$ per day (ANCOVA, $p=0.005$ ).

Table 2 shows WBC counts according to the total number of seven healthy practices, the distribution of which differed significantly between men and women $(p=0.001)$. The category occupied by the largest number was ' 5 healthy practices' in men $(381 / 1,492,25.5 \%)$ and in women $(103 / 316,32.6 \%)$. Each proportion of current smoker and obese were decreased along with the increase total number of seven healthy practices. Crude and ageadjusted WBC counts decreased significantly along with an increase in healthy practices among both men and women $(p<0.001$, Trend test).

All subjects were classified into four groups by the total number of healthy practices, that is, 0 to $3,4,5$, and 6 to 7 , since only a few subjects had a lower or higher total number of healthy practices (Table3). In men, age-adjusted WBC counts decreased along with the progress of categories in the order of the increase in healthy practices, which were $5,782,5,397,5,182$, and 5,013 in ' $0-3$ ', ' 4 ', ' 5 ', and ' $6-7$ ' healthy practices, respectively $(p<0.001$, Trend test). In women, the respective value was 5,147, $4,752,5,021$, and $4,722(p=0.13$, Trend test), respectively.

When the total number of healthy practices was used as a continuous variable in the linear regression model to estimate the magnitude of their effect on WBC counts, after adjustment for age, WBC counts decreased significantly by $204.9 \pm 23.7$ cells $/ \mu$ l (means \pm SE) and $117.6 \pm 53.2 \mathrm{cells} / \mu$ l for an increase in one healthy practice among men and women $(p<0.05)$, respectively. In addition, to exclude the effect of smoking status and obesity, we entered the total number of healthy practices except for smoking and obesity (0-5) as a continuous variable in the linear regression model. After adjustment for age, WBC counts decreased significantly by $101.3 \pm 30.0 \mathrm{cells} / \mu \mathrm{l}(p=0.001)$ in men and $27.0 \pm 60.5$ cells $/ \mu \mathrm{l}(p=0.66)$ in women for an increase in one healthy practice, respectively. In sub analyses, the association between WBC counts and unhealthy practices (obese and smoking) was assessed by multiple linear regression analysis, age adjusted WBC counts was positively associated with obese and current smoking (standardized $\beta$ for obese and current smoking: $0.13, p<0.001$ and 0.31 $p<0.001$, Total $\mathrm{R}^{2}: 0.35$ in men, $0.18, p<0.001$ and 0.20 $p<0.001$, Total $\mathrm{R}^{2}: 0.30$ in women, respectively).

Additional statistical analyses were done by excluding individuals (146 men, 20 women) with a medical history of heart disease, stroke, diabetes or cancer did not alter the results (data not shown).

\section{Discussion}

We found an independent negative association between WBC counts and healthy practices such as normal weight (BMI of $<25 \mathrm{~kg} / \mathrm{m}^{2}$ ), and current non-smoking. On the other hand, men with a daily ethanol intake of $23 \mathrm{~g}$ and over had significantly lower WBC counts. The total number of healthy practices was significantly and negatively associated with WBC counts among both men and women. To our knowledge, this is the first report suggesting that cultivating healthier practices would lead to lower WBC counts, or the prevention of low-grade inflammation.

Obesity and current smoking were found to be independently associated with elevated WBC counts, both of which are consistent with previous reports ${ }^{9-11,15-17)}$. In addition, higher cigarette consumption (more than 20 cigarettes per day) was positively associated with WBC counts in men. Although the precise mechanisms underlying the association between these two health practices and WBC counts have not been clearly defined, changes in secreted amount of proinflammatory cytokines such as tumor necrosis factor-alpha (TNF- $\alpha$ ) and interleukin-6 (IL-6), which are known to increase WBC counts ${ }^{1}$, may be related to our findings. These cytokines are secreted by adipocytes and expressed at higher levels in the adipose tissue of obese subjects ${ }^{18)}$. Moreover, a direct injury to epithelial and endothelial surfaces known to have an inflammatory impact on lung tissue and/or changes in the level of cytokines such as IL- 6 caused by the constituents of cigarette smoke may induce elevations in the peripheral WBC count and modifications in leukocyte function $^{19)}$.

On the contrary, ethanol intakes of $23 \mathrm{~g}$ and over per day were associated with reduced WBC counts than those with none or less than $23 \mathrm{~g}$ in men, and non-drinkers had higher WBC counts than those with drinkers. As for the effect of alcohol intake on systemic markers of inflammation, Nakanishi et al. ${ }^{20)}$ found a negative dose-response relationship between alcohol consumption and WBC counts after adjustment for BMI and cardiovascular risk factors such as systolic blood pressure, total cholesterol, and fasting plasma glucose. Imhof et al. ${ }^{21)}$ also reported a U-shaped association between alcohol intake and Creactive protein (CRP) with a negative peak at alcohol intake of $40-60 \mathrm{~g}$ ethanol per day. The suppression of production of the TNF- $\alpha$ and IL- 6 and/or the inhibition of their action caused by alcohol consumption are proposed as possible mechanisms. Although we could not evaluate the effect of heavy alcohol intake on WBC counts because of the small number of heavy drinkers, moderate or appropriate level of alcohol intake may have some favorable effects in this regard. 
The other four health practices such as perceived mental stress, paying attention to eating habits, sleep, and physical activity showed no significant association with individual WBC counts. Although not all good healthy practices were associated with lower WBC counts, the clustering of good practices within individuals as measured by the number of healthy practices was significantly and linearly associated with low WBC counts in both men and women. An increase in one healthy practice decreased WBC counts by $204.9 \pm 23.7$ cells $/ \mu$ l and $117.6 \pm 53.2$ cells $/ \mu$ l among men and women, respectively. This finding indicates that the total number of selected 7 health practices may reflect the magnitude of lowgrade inflammation accurately beyond their mere addition. This phenomenon is maybe due to other health practices accompanying each of 7 health practices.

It was considered that smokers or obese were probably to have smaller healthy practices, indeed, the proportion of current smoker and obese were decreased along with the increase with total number of healthy practices. Although, the total number of healthy practices except for smoking and obesity was also negatively associated with WBC counts in men, this may imply not only smoking status and obesity but also other five health practices was associated with WBC counts.

Several limitations of this study deserve mention. First, the directionality of associations could not be conclusively established because the study is cross-sectional. Second, only one WBC count measurement was performed, whereas multiple WBC measurements over time and their changes may provide more accurate and detailed information on the association between lifestyle and WBC counts. Third, we used self-reported questionnaires to obtain data on individual health practices. In terms of the validity of study variables, self-reported health practices may influence the observed results, thus causing us to underestimate the true association. Finally, the subjects in this study were comprised of middle-aged Japanese men and women, and thus the results may not be applicable to Westerners with different lifestyles and body sizes. Breslow et al. ${ }^{14)}$ proposed seven health practices including "not eating between meals" and "having breakfast" as preventive factors for higher mortality, which were somewhat different from those we selected. We chose one variable as eating behavior whether subjects usually pay attention to eating habits or not, since we could not obtain data about "eating between meals or not", and more than $90 \%$ of men and women who pay attention to eating habits had breakfast almost every day. Their observation may be in part explained by elevated WBC count.

In summary, this cross-sectional study on the association of health practices with WBC counts confirmed previously reported studies and provided additional insights to prevent the development of low-grade inflammation. Among fundamental health practices studied, obesity and cigarette smoking were more closely associated with elevated WBC counts. On the other hand, we found a strong association between reduced WBC counts and a number of healthy practices. We strongly recommended efforts to modify unhealthy practices one by one and to maintain healthy ones as primary elements in any strategy for the prevention of atherosclerotic diseases, in addition, to quit smoking or abstain from heavy smoking especially in men was important to prevent the low-grade inflammation.

\section{Acknowledgements}

This work was supported in part by grants to Rei Otsuka from the Japan Society for the Promotion of Science and to Hideaki Toyoshima (17390185), Koji Tamakoshi (18590594), and Hiroshi Yatsuya (17790384) from the Japanese Ministry of Education, Culture, Sports, Science and Technology, the Kato Memorial Trust for Nambyo Research, and the Japan Atherosclerosis Prevention Fund (JAPF).

\section{References}

1) Hoffman M, Blum A, Baruch R, Kaplan E, Benjamin M (2004) Leukocytes and coronary heart disease. Atherosclerosis 172, 1-6.

2) Nagasawa N, Tamakoshi K, Yatsuya H, Hori Y, Ishikawa M, Murata C, Zhang H, Wada K, Otsuka R, Mabuchi T, Kondo T, Toyoshima H (2004) Association of white blood cell count and clustered components of metabolic syndrome in Japanese men. Circ J 68, 892-7.

3) Margolis KL, Manson JE, Greenland P, Rodabough RJ, Bray PF, Safford M, Grimm RH Jr, Howard BV, Assaf AR, Prentice R; Women's Health Initiative Research Group (2005) Leukocyte count as a predictor of cardiovascular events and mortality in postmenopausal women: the Women's Health Initiative Observational Study. Arch Intern Med 165, 500-8.

4) Brown DW, Giles WH, Croft JB (2001) White blood cell count: an independent predictor of coronary heart disease mortality among a national cohort. J Clin Epidemiol 54, 316-22.

5) Kannel WB, Anderson K, Wilson PW (1992) White blood cell count and cardiovascular disease. Insights from the Framingham Study. JAMA 267, 1253-6.

6) Sakuta H, Suzuki T, Yasuda H, Ito T (2005) White blood cell count is associated with plasma total homocysteine in Japanese men. Scand J Clin Lab Invest 65, 447-52.

7) Sakuta H, Suzuki T (2006) Physical activity and selected cardiovascular risk factors in middle-aged male personnel of self-defense forces. Ind Health 44, 184-9. 
8) Pitsavos C, Panagiotakos DB, Chrysohoou C, Kavouras S, Stefanadis C (2005) The associations between physical activity, inflammation, and coagulation markers, in people with metabolic syndrome: the ATTICA study. Eur J Cardiovasc Prev Rehabil 12, 151-8.

9) Nishitani N, Sakakibara H (2007) Subjective poor sleep and white blood cell count in male Japanese workers. Ind Health 45, 296-300.

10) Abel GA, Hays JT, Decker PA, Croghan GA, Kuter DJ, Rigotti NA (2005) Effects of biochemically confirmed smoking cessation on white blood cell count. Mayo Clin Proc 80, 1022-8.

11) Zalokar JB, Richard JL, Claude JR (1981) Leukocyte count, smoking, and myocardial infarction. N Engl J Med 304, 465-8.

12) Nakanishi N, Suzuki K, Tatara K (2003) Association between lifestyle and white blood cell count: a study of Japanese male office workers. Occup Med (Lond) 53, $135-7$.

13) Hansen LK, Grimm RH Jr, Neaton JD (1990) The relationship of white blood cell count to other cardiovascular risk factors. Int J Epidemiol 19, 881-8.

14) Breslow L, Breslow N (1993) Health practices and disability: some evidence from Alameda County. Prev Med 22, 86-95.

15) Examination Committee of Criteria for 'Obesity Disease' in Japan; Japan Society for the Study of Obesity (2002) New criteria for 'obesity disease' in Japan. Circ J 66, 987-92.

16) Saito I, Yonemasu K, Inami F (2003) Association of body mass index, body fat, and weight gain with inflammation markers among rural residents in Japan. Circ J 67, 323-9.

17) Mabuchi $T$, Yatsuya $H$, Tamakoshi K, Otsuka R, Nagasawa N, Zhang H, Murata C, Wada K, Ishikawa M, Hori Y, Kondo T, Hashimoto S, Toyoshima H (2005) Association between serum leptin concentration and white blood cell count in middle-aged Japanese men and women. Diabetes Metab Res Rev 21, 441-7.

18) Yudkin JS, Stehouwer CD, Emeis JJ, Coppack SW (1999) C-reactive protein in healthy subjects: associations with obesity, insulin resistance, and endothelial dysfunction: a potential role for cytokines originating from adipose tissue? Arterioscler Thromb Vasc Biol 19, $972-8$.

19) McCarty MF (1999) Interleukin-6 as a central mediator of cardiovascular risk associated with chronic inflammation, smoking, diabetes, and visceral obesity: downregulation with essential fatty acids, ethanol and pentoxifylline. Med Hypotheses 52, 465-77.

20) Nakanishi N, Yoshida H, Okamoto M, Matsuo $Y$, Suzuki K, Tatara K (2003) Association of alcohol consumption with white blood cell count: a study of Japanese male office workers. J Intern Med 253, 367-74.

21) Imhof $A$, Froehlich $M$, Brenner H, Boeing H, Pepys MB, Koenig W (2001) Effect of alcohol consumption on systemic markers of inflammation. Lancet 357, $763-7$. 